\title{
Suxiao Jiuxin Pill Induces Potent Relaxation and Inhibition on Contraction in Human Artery and the Mechanism
}

\author{
Xiao-Yan Bai, ${ }^{1}$ Ping Zhang, ${ }^{2}$ Qin Yang, ${ }^{1,3}$ Xiao-Cheng Liu, ${ }^{4}$ Jun Wang, ${ }^{4}$ Yong-Ling Tong, \\ Song-Jin Xiong, ${ }^{5}$ Li-Hua Liu, ${ }^{2}$ Lei Wang, ${ }^{5}$ and Guo-Wei He ${ }^{1,6}$ \\ ${ }^{1}$ TEDA International Cardiovascular Hospital \& The Affiliated Hospital of Hangzhou Normal University, Tianjin \& Hangzhou, China \\ ${ }^{2}$ Tianjin Zhongxin Pharmaceutical Group Co., Ltd. No. 6, Traditional Chinese Medicine Factory, Tianjin, China \\ ${ }^{3}$ The Chinese University of Hong Kong, Hong Kong \\ ${ }^{4}$ TEDA International Cardiovascular Hospital, Tianjin, China \\ ${ }^{5}$ TEDA School of Biological Sciences and Biotechnology, Nankai University, Tianjin, China \\ ${ }^{6}$ Department of Surgery, Oregon Health and Science University, Portland, OR, USA
}

Correspondence should be addressed to Guo-Wei He; gwhezj@163.com

Received 13 August 2013; Accepted 11 February 2014; Published 7 April 2014

Academic Editor: Hong Zhang

Copyright (C) 2014 Xiao-Yan Bai et al. This is an open access article distributed under the Creative Commons Attribution License, which permits unrestricted use, distribution, and reproduction in any medium, provided the original work is properly cited.

Suxiao Jiuxin Pill, a compound Chinese traditional medicine with main components of tetramethylpyrazine and borneol, is widely used for antiangina treatment in China but its pharmacological effect on human blood vessels is unknown. We investigated the effect and possible mechanism of SJP in the human internal mammary artery (IMA, $n=78$ ) taken from patients undergoing coronary surgery. SJP caused full relaxation in KCl- $(99.4 \pm 10.5 \%, n=6)$ and U46619- $(99.9 \pm 5.6 \%, n=6)$ contracted IMA. Pretreatment of IMA with plasma concentrations of SJP $(1 \mathrm{mg} / \mathrm{mL})$, calculated from the plasma concentration of its major component borneol, significantly depressed the maximal contraction to $\mathrm{KCl}$ (from $35.8 \pm 6.0 \mathrm{mN}$ to $12.6 \pm 5.6 \mathrm{mN}, P=0.03$ ) and U46619 (from $19.4 \pm 2.9 \mathrm{mN}$ to $5.7 \pm 2.4 \mathrm{mN}, P=0.007)$ while SJP at $10 \mathrm{mg} / \mathrm{mL}$ abolished the subsequent contraction. Endothelium denudation and inhibition of eNOS significantly altered the SJP-induced relaxation without changes of eNOS expression. We conclude that SJP has a potent inhibitory effect on the vasoconstriction mediated by a variety of vasoconstrictors in human arteries. The vasorelaxation involves both endothelium-dependent and -independent mechanisms. Thus, the effect of SJP on human arteries demonstrated in this study may prove to be particularly important in vasorelaxing therapy in cardiovascular disease.

\section{Introduction}

Cardiovascular disease (CVD), particularly coronary heart disease (CHD), remains a major cause of mortality, morbidity, and disability in the US and other Westernized societies [1] as well as in low-income and middle-income countries [2]. A Chinese traditional medicine pill named Suxiao JiuxinWan (Suxiao Jiuxin Pill) has been widely used for treatment of cardiovascular diseases in China on its own or in conjunction with conventional western treatments for angina pectoris, such as with beta blockers, calcium channel blockers, and nitrates (glyceryl trinitrate, GTN) [3, 4]. In fact, Suxiao Jiuxin Pill has been added on the national essential drug list of China for the treatment of cardiocerebral vascular diseases $[5,6]$. Suxiao Jiuxin Pill has been shown to cause remission of angina pectoris [7], improve anginal symptoms, and reduce the use of GTN — a drug used to quickly relieve angina symptoms [8].

Suxiao Jiuxin Pill is a compound Chinese traditional medicine. The main components of Suxiao Jiuxin Pill include the major components extracted from Ligusticum Chuanxiong Hort, that is, tetramethylpyrazine (TMP, Ligustrazine, or Chuan-Xiong-Qin), and borneol (Borneolum Syntheticum or Bing Pian). The average recoveries of TMP and borneol were $87.38 \%$ and $97.32 \%$ [9]. In addition, five metal elements including calcium, magnesium, iron, copper, and zinc in Suxiao Jiuxin Pill are comparatively rich [10].

TMP is the biologically active ingredient isolated from a popular Chinese medicinal plant Ligusticum Chuanxiong 
Hort (Szechwan Lovage Rhizome, or Chuan-Xiong) that is a perennial Chinese herb, with the effect of blood circulation of Qi, chills and pain relief, and gloomy dampness in traditional Chinese medicine for thousands of years [11].

TMP has been synthesized and used widely in oriental medicine since the 1970 s to effectively treat several cardiovascular diseases including ischemic heart disease, cerebrovascular and thrombotic vascular diseases [12]. The pharmacological effect of TMP is well established including alleviating kidney and brain damage induced by ischemia perfusion in rats via scavenging oxygen-free radicals $[13,14]$, significantly suppressing oxidative stress, and attenuating cell death in neuronal cultures induced by glutamate analogue and ironmediated oxidative stress [15-18].

Borneol is a bicyclic organic compound and a terpene. The hydroxyl group in this compound is placed in an endoposition. Borneol is easily oxidized to the ketone (camphor). One historical name for borneol is Borneo camphor which explains the name [19]. Cinnamomum camphora is native to south of the Yangtze River of China, Taiwan, Japan, Korea, and Vietnam and has been introduced to many other countries [20].

Borneol is used in traditional Chinese medicine as moxa. In modern era, it is one of the active compounds of the HeartProtecting Musk Pellets [21]. An early description is found in the Bencao Gangmu (Compendium of Materia Medica or BenCao-Gang-Mu) published in 1590 by Li Shi-Zhen.

Despite the above-mentioned and well-known effect of these compounds, the pharmacological effect of Suxiao Jiuxin Pill on the human vessels is not reported and this would provide important information as to the clinical use of Suxiao Jiuxin Pill in patients with coronary artery disease.

It is well known now that coronary artery bypass grafting (CABG) surgery is the effective therapeutic method for coronary artery disease and the internal mammary artery (IMA) is the most commonly used arterial graft due to its superior graft patency and increased long-term survival [2225]. Graft spasm of the IMA is a long recognized problem $[23,26-28]$. There have been extensive investigations on the antispastic therapy in the past decades to solve this problem and such efforts have greatly reduced the problem of spasm in arterial grafts [22, 29-32]. However, spasm of the IMA may occur even in the current practice as recently reported $[33,34]$. The mechanism of graft vasospasm is a complex physiological and pathophysiological issue and may involve mechanical, physical, and pharmacological stimulation. Therefore, reversal and prevention of graft vasospasm would be a challenging task for a long time.

Further, as is well known now, the vascular endothelium plays a crucial role in the maintenance of vascular tone and nitric oxide (NO) produced by endothelial NO synthase (eNOS) is required for normal vascular function. Alteration of NO synthesis has been recognized as a common mechanism by which several cardiovascular risk factors mediate certain deleterious effect on the endothelium.

Taken together, the present study was designed to investigate the vasorelaxation induced by Suxiao Jiuxin Pill and its inhibitory effects on vasoconstriction mediated by various vasoconstrictors as well as protein expressions of eNOS in the human IMA.

\section{Material and Methods}

2.1. Vessels Preparation. Human IMA ( $n=78$, from 30 patients) segments were obtained from patients undergoing CABG. Approval to use discarded IMA tissue was given by the Institutional Review Board of TEDA International Cardiovascular Hospital with patient consent. The details of the procedure were reported before [29, 35-38]. During CABG, using IMA grafts, the discarded distal end was carefully removed and immediately placed in a container with oxygenated physiological salt solution maintained at $4^{\circ} \mathrm{C}$ and transferred to the laboratory within 5-10 minutes. After the adherent connective tissue was carefully disposed, the IMA was precisely cut into $3 \mathrm{~mm}$ long segments. The Krebs solution was composed of (in $\mathrm{mM}$ ) $\mathrm{Na}^{+} 144, \mathrm{~K}^{+} 5.9, \mathrm{Ca}^{2+} 2.5$, $\mathrm{Mg}^{2+} 1.2, \mathrm{Cl}^{-} 128.7, \mathrm{HCO}_{3}{ }^{-} 25, \mathrm{SO}_{4}{ }^{2-} 1.2, \mathrm{H}_{2} \mathrm{PO}_{4}{ }^{-} 1.2$, and glucose 11 , which was aerated with a gas mixture of $95 \% \mathrm{O}_{2}$ and $5 \% \mathrm{CO}_{2}$ at $37^{\circ} \mathrm{C} \pm 0.1^{\circ} \mathrm{C}$ during the period of experiment.

2.2. Organ Bath Techniques. Human IMA ring segments were suspended on wire hooks in a $10 \mathrm{~mL}$ bath on a myograph modified for large vessel studies (Model 610M, DMT Company, Denmark). Each ring segment unstretched on the wire hooks was equilibrated in Krebs solution for at least 30 minutes.

Normalization. The organ bath technique described previously in detail was used in this study [29, 39, 40]. Briefly, each ring segment was stretched up in progressive steps every minute to determine the individual length-tension curve. A computer iterative fitting program (Myodaq and Myodata, version 2.01, Maastricht University, The Netherlands) was used to determine the exponential curve, pressure, and the internal diameter. At the end of each step, the internal diameter $(\mu \mathrm{m})$ and the corresponding wall tension $\left(\mathrm{mN} / \mathrm{mm}^{2}\right)$ were recorded. When the transmural pressure on the rings reached $100 \mathrm{mmHg}$, as determined according to the length-tension curves, the stretch-up procedure was stopped and the rings were released to $90 \%$ of their internal circumference at $100 \mathrm{mmHg}$. This degree of passive tension was then maintained throughout the experiment.

2.3. Pharmacological Protocols. After normalization, the artery rings were equilibrated for at least 45 minutes.

2.4. Relaxation by Suxiao Jiuxin Pill in KCl- and U46619Induced Contraction. Cumulative concentration- (-2 1.25 $\log \mathrm{mg} / \mathrm{mL}$ ) relaxation curve for Suxiao Jiuxin Pill was established in IMA rings precontracted with $\mathrm{K}^{+}(\mathrm{KCl} 25 \sim$ $40 \mathrm{mM}, n=6)$ or U46619 (10 20 nM, $n=6)$. The concentration of the vasoconstrictors $\mathrm{K}^{+}$[29] or U46619 [38, 39] was determined on the basis of the previous studies. Only one concentration-relaxation curve was obtained from each IMA ring. To allow each concentration of Suxiao Jiuxin Pill to reach the relaxing plateau, usually $10 \sim 30 \mathrm{~min}$ was 
given before the next dose to be added. Relaxations to Suxiao Jiuxin Pill $(-2 \sim 1.25 \log \mathrm{mg} / \mathrm{mL})$ were recorded and expressed as a percentage of the vasoconstrictor-induced precontraction. Glyceryl trinitrate- (GTN) and amlodipine(both at $-10-4.5 \log M$ ) induced relaxations were used in comparison to Suxiao Jiuxin Pill.

\subsection{Depression of Contraction by Pretreatment with Suxiao} Jiuxin Pill in IMA. After equilibration for at least $60 \mathrm{~min}$, IMA rings were incubated with Suxiao Jiuxin Pill (Suxiao Jiuxin Pill $1 \mathrm{mg} / \mathrm{mL}, 10 \mathrm{mg} / \mathrm{mL}$, or vehicle for $30 \mathrm{~min}$ ). The concentration of Suxiao Jiuxin Pill was chosen from the plasma concentration, calculated from the plasma concentration of its major component borneol in the human reported previously [41, 42]. The vehicle ethanol was added in the control group to exclude the effect of ethanol that was used as the solvent of Suxiao Jiuxin Pill. In each experiment, one of the two vasoconstrictors was added to construct the cumulative concentration-contraction curve. The cumulative concentration-contraction curve was established for $\mathrm{KCl}$ (5 $120 \mathrm{mM})$ and $\mathrm{U} 46619(-10 \sim-6 \log M)$, respectively. Only one concentration-contraction curve was obtained from each ring. Data regenerated from our laboratories on the inhibitory effects by GTN- [29] and amlodipine- [43] (both at their own plasma concentrations) induced relaxations were used in comparison to Suxiao Jiuxin Pill.

2.6. Endothelium-Dependent Relaxation. To investigate the role of endothelium denudation and endothelium-derived NO and eNOS, Suxiao Jiuxin Pill- $(-2 \sim 1.25 \log \mathrm{mg} / \mathrm{mL})$ induced relaxation in $40 \mathrm{mM} \mathrm{KCl}$-induced precontraction was examined in endothelium-denuded and eNOS inhibitor LNNA $(300 \mu \mathrm{M})$ pretreated IMA rings. The endotheliumintact rings were pretreated with L-NNA for $60 \mathrm{~min}$ before adding the $\mathrm{KCl}$. Endothelium-intact IMA rings from the same patient were studied as control.

In the endothelium-denuded IMA rings, the endothelium was removed by gently rubbing the intima with a paper stick [36]. We have repeatedly demonstrated that this method may successfully remove the endothelium and eliminate the endothelium-dependent relaxation. In fact, this method can abolish the acetylcholine-induced endothelium-dependent relaxation as we recently published in the human IMA [44], indicating that the endothelium is successfully removed.

2.7. Western Blot Analysis of eNOS. IMA samples were homogenized in lysis buffer (KeyGEN, Inc., Nanjing, China), and the lysates were incubated in ice for 1 hour followed by 10 minutes of centrifugation at $10,000 \mathrm{rpm}$. After the sample was heated at $100^{\circ} \mathrm{C}$ for 5 minutes to denature it, $120 \mathrm{mg}$ protein for each sample was separated by $8 \%$ polyacrylamide gel electrophoresis (Page Gel, Inc., San Diego, CA) together with the prestained protein ladder (MBI Fermentas, Inc.). The proteins were transferred electrophoretically to the polyvinylidene fluoride membrane (Millipore, Billerica, Mass). The membrane was blocked with blocking buffer (trisbuffered saline solution, $0.1 \%$ polysorbate $20,5 \%$ nonfat dry milk) for 3 hours at room temperature and incubated with primary antibody against eNOS (1:1000; Cell Signaling Technology, Inc., Boulder, Colo) overnight at $4^{\circ} \mathrm{C}$. Equivalent protein on the same lane was confirmed by stripping and reblotting with glyceraldehyde 3-phosphate dehydrogenase (1:1000; Cell Signaling Technology). The secondary goat anti-rabbit antibody conjugated to horseradish peroxidase (Santa Cruz Biotechnology, Inc., Santa Cruz, CA) at a dilution of 1:5000 was added the next day. Finally, blots were developed with an enhanced chemiluminescence detection system (Amersham Pharmacia ECL reagents; GE Healthcare Biosciences, Piscataway, NJ) and exposed to X-ray films. The protein bands were quantified with QuantityOne software (Bio-Rad Laboratories, Inc., Hercules, CA), normalized by glyceraldehyde 3-phosphate dehydrogenase and expressed as multiples of control. These methods are well used in our laboratories $[45,46]$.

2.8. Data Analysis. The sensitivity of an agent was expressed as $\mathrm{EC}_{50}$, the effective concentration that caused $50 \%$ of maximal relaxation or contraction. The $\mathrm{EC}_{50}$ was determined from each concentration-relaxation curve by a sigmoid logistic curve-fitting equation: $E=M A^{P} /\left(A^{P}+K^{P}\right)$, where $E$ is response, $M$ is maximal relaxation, $A$ is concentration, $K$ is $\mathrm{EC}_{50}$ concentration, and $P$ is the slope parameter [39]. A computerized program was used for the curve fitting.

All results were expressed as mean \pm SEM. Statistical comparisons of the cumulative responses of relaxation or contraction under different treatments were performed by two-way analysis of variance (ANOVA) with repeated measures (SPSS, Inc., Chicago, IL), which was followed by a post hoc Bonferroni test to detect the individual differences. $P<$ 0.05 was considered to be statistically significant.

2.9. Materials. Chemicals involved in this study were potassium chloride, U46619 (Cayman Chemical, Ann Arbor, MI), L-NNA (Sigma, St. Louis, MO), and Suxiao Jiuxin Pill (Tianjin Zhongxin Pharmaceutical Group Co., Ltd. China).

Suxiao Jiuxin Pill was diluted in $75 \%$ ethanol to $300 \mathrm{mg} / \mathrm{mL}$ and $100 \mathrm{mg} / \mathrm{mL}$ and diluted in $30 \%$ ethanol to $10 \mathrm{mg} / \mathrm{mL}$. Stock solution of U46619 was kept frozen until required. Suxiao Jiuxin Pill was stored at room temperature.

\section{Results}

3.1. Resting Parameters of IMA Ring Segments. The internal diameter of the 60 IMA ring segments at an equivalent transmural pressure of $100 \mathrm{mmHg}$ (D100) was $1.87 \pm 0.08 \mathrm{~cm}$ as determined in the normalization procedure. When the IMA ring segments were set at a resting diameter of $0.9 \mathrm{D} 100$, the equivalent transmural pressure was $90.3 \pm 0.5 \mathrm{mmHg}$, and the resting force was $16.1 \pm 1.6 \mathrm{mN}$.

3.2. Similar Relaxation Effect of Suxiao Jiuxin Pill in KCl-and U46619-Induced Contraction. In the relaxation studies, the precontraction was $16.0 \pm 2.3 \mathrm{mN}$ by $\mathrm{KCl}$ and $16.6 \pm 5.2 \mathrm{mN}$ by $\mathrm{U} 46619$ ( $P>0.05 ; n=12$ in each group).

Suxiao Jiuxin Pill caused full relaxation in $\mathrm{KCl}-$ and U46619-precontracted IMA rings (99.4 $\pm 10.5 \%$ for $\mathrm{KCl} ; 99.9 \pm$ 

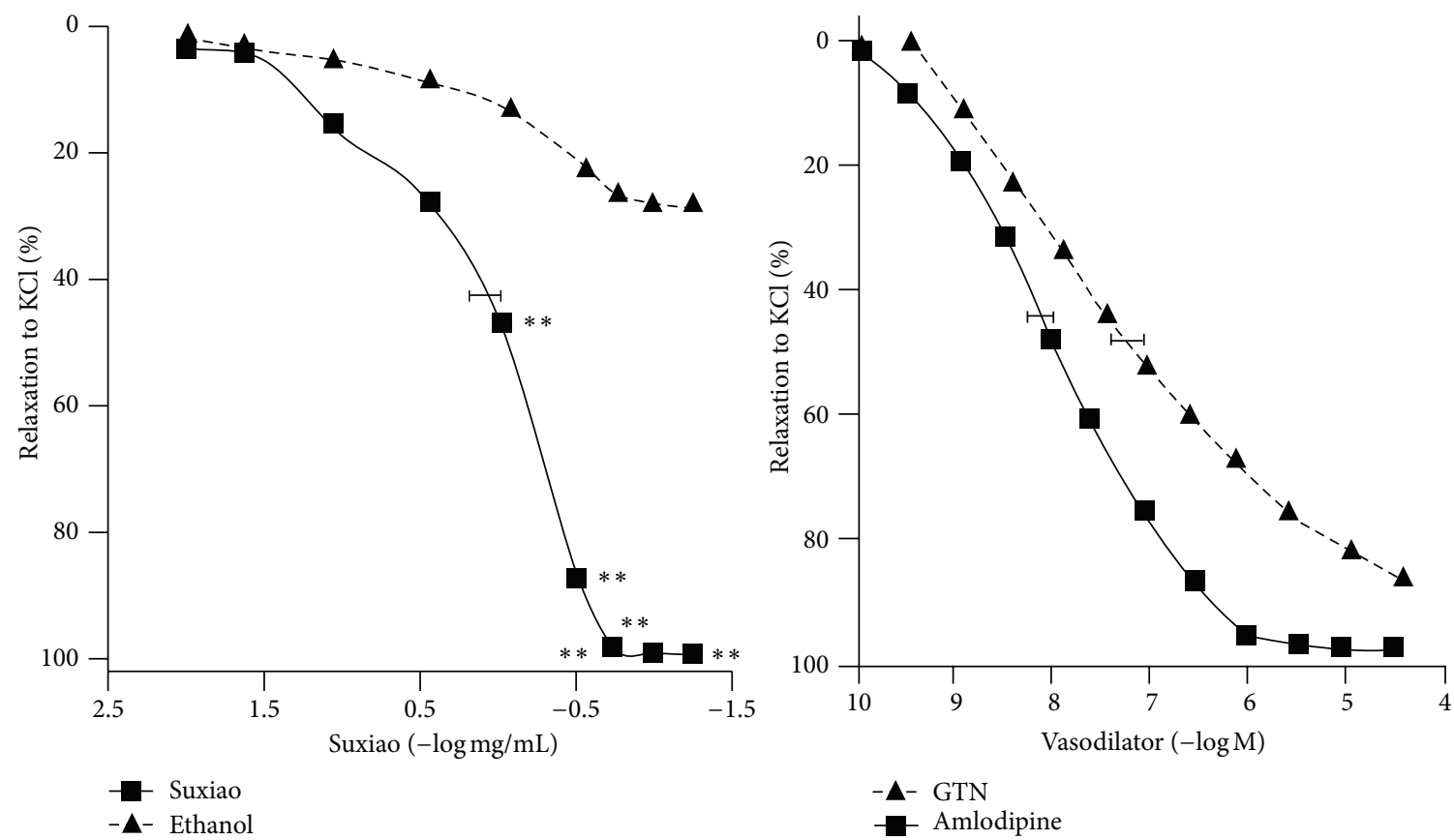

(a)
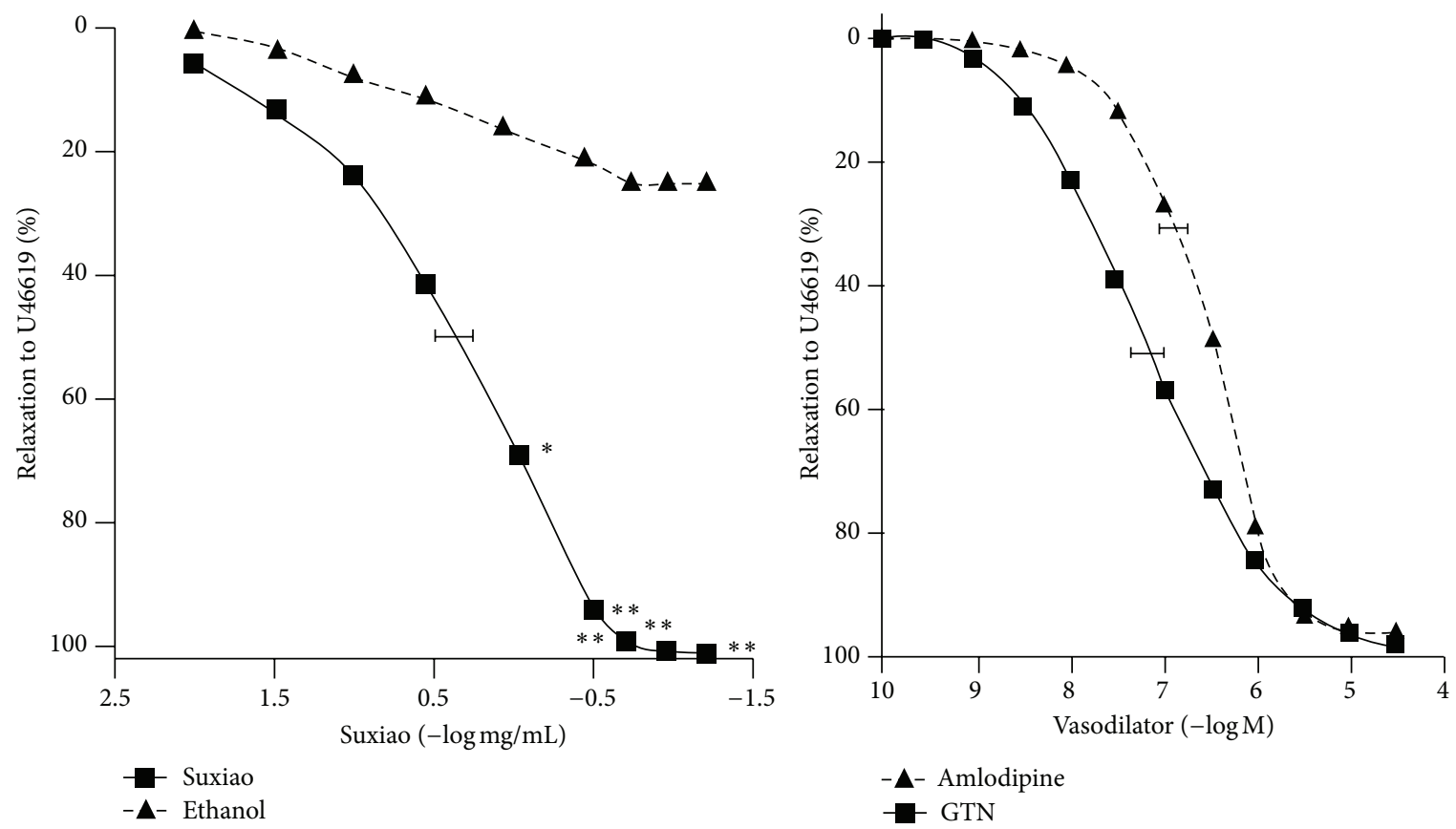

(b)

FIgURe 1: Mean concentration- (log mg/mL for Suxiao Jiuxin Pill; - $\log M$ for GTN and amlodipine) relaxation curves for Suxiao Jiuxin Pill, GTN, and amlodipine in KCl- (25 40 mM, (a)) or U46619- (10 20 nM, (b)) induced contraction in human IMA ( $n=8$ in each group). \% relaxation to $\mathrm{KCl}$ (or U46619): percentage relaxation induced by Suxiao or other vasodilators in the KCl- (or U46619-) induced precontraction. Ethanol was the solvent of Suxiao Jiuxin Pill. Values are expressed as mean \pm SEM. ${ }^{*} P<0.05,{ }^{* *} P<0.01$ compared to vehicle group (unpaired $t$-test). KCl: potassium chloride; GTN: glyceryl trinitrate; Suxiao: Suxiao Jiuxin Pill.

$5.6 \%$ for U46619; $n=6$ in each group). Two-way ANOVA for all concentrations revealed that there were no differences ( $P=0.43$; 95\% CI: 32.0, 67.8\%) between KCl- and U46619precontraction. The relaxation of Suxiao Jiuxin Pill was with similar potency to $\mathrm{KCl}$ and $\mathrm{U} 46619\left(\mathrm{EC}_{50}\right.$ : $-0.15 \pm 0.23$ versus $-0.19 \pm 0.21 \log \mathrm{mg} / \mathrm{mL}, P>0.05$; unpaired $t$-test) (Figure 1). Importantly, two-way ANOVA for all concentrations detected that there were significant differences of relaxation caused by Suxiao Jiuxin Pill compared with the control $(P=0.002$; 95\% CI: 29.1, 52.0\% for KCl-precontraction and 


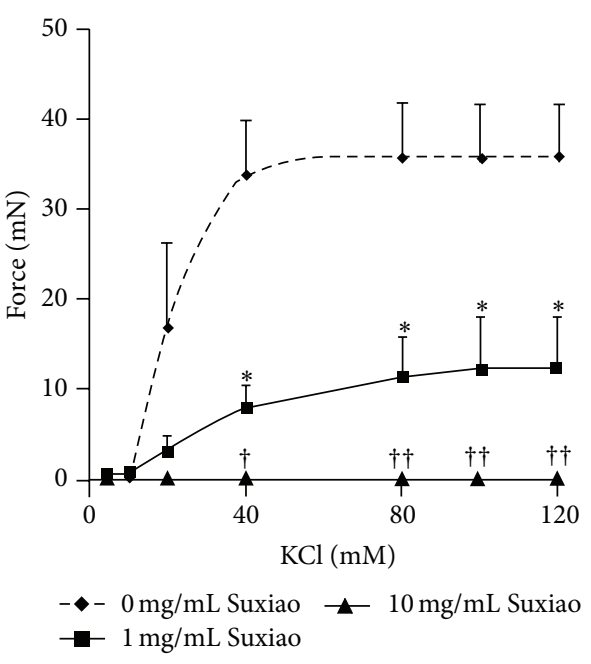

(a)

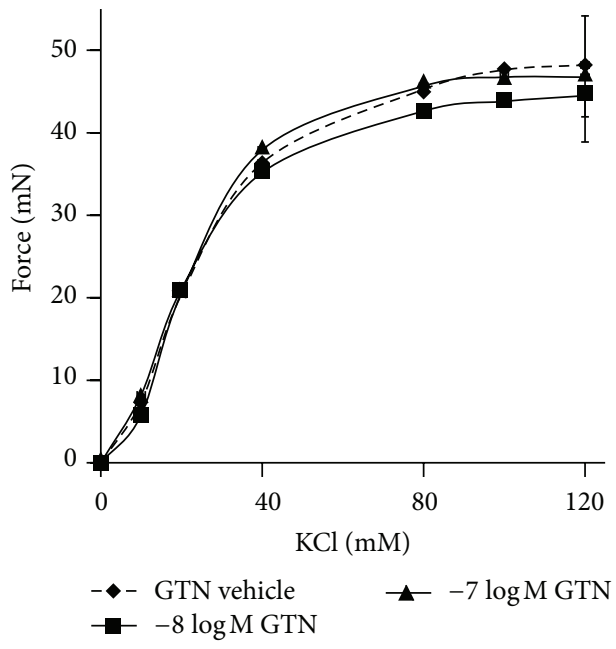

(b)

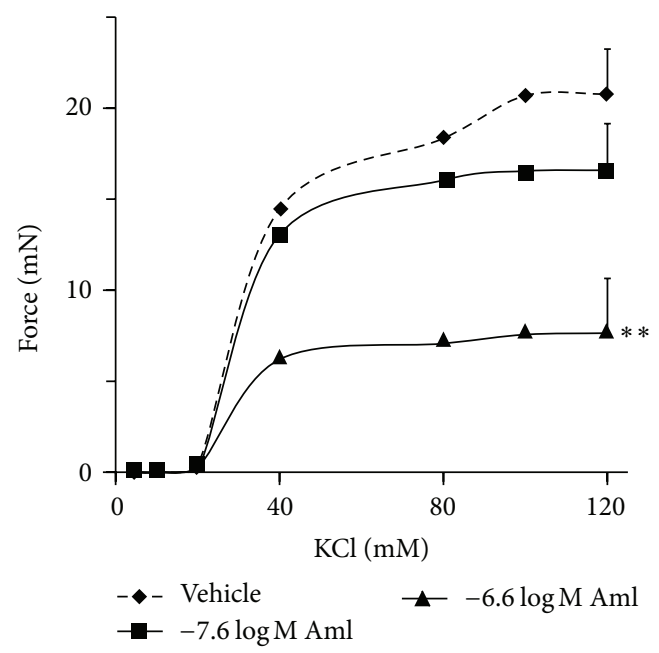

(c)

Figure 2: Mean concentration- $(\mathrm{mM})$ contraction $(\mathrm{mN})$ curves for $\mathrm{KCl}$ when Suxiao Jiuxin Pill $1 \mathrm{mg} / \mathrm{mL}(\boldsymbol{\bullet}), 10 \mathrm{mg} / \mathrm{mL}(\mathbf{\Delta})$, or vehicle (\);

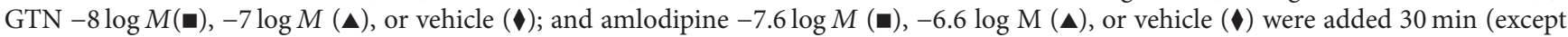
for GTN were added $10 \mathrm{~min}$ ) before the contraction started in the human IMA ring segments ( $n=6$ in each group). Three rings from an IMA segment taken from the same patient were allocated into each group. Values are expressed as mean \pm SEM. ${ }^{*} P<0.05$ compared to vehicle group in $1 \mathrm{mg} / \mathrm{mL}$ Suxiao Jiuxin Pill pretreatment; ${ }^{\dagger} P<0.05,{ }^{\dagger \dagger} P<0.01$ compared to vehicle group in $10 \mathrm{mg} / \mathrm{mL}$ Suxiao Jiuxin Pill pretreatment; ${ }^{* *} P<0.01$ compared to the control (vehicle) in $-6.6 \log M$ amlodipine pretreatment (unpaired $t$-test). $P=0.016$ between the treatment of $-6.6 \log M$ amlodipine and the control (vehicle) in KCl-contracted groups. KCl: potassium chloride; GTN: glyceryl trinitrate; Suxiao: Suxiao Jiuxin Pill; Aml: amlodipine.

$P=0.014$; 95\% CI: 29.9, 69.8\% for U46619-precontraction); there were no differences of relaxation caused by Suxiao Jiuxin Pill between $\mathrm{KCl}$ - and U46619-precontraction $(P=$ 0.43; 95\% CI: 22.6, 58.5\%).

3.3. Depression of Contraction by Pretreatment with Suxiao Jiuxin Pill in IMA. The magnitude of the maximal contraction to $\mathrm{KCl}$ was significantly depressed in the group pretreated with plasma concentration of Suxiao Jiuxin Pill $(1 \mathrm{mg} / \mathrm{mL})$, calculated from the plasma concentration of its major component borneol $(P=0.03$ to control; $95 \%$
CI: $3.1,43.2 \mathrm{mN}$; unpaired $t$-test). Two-way ANOVA for all concentrations also demonstrated a significant difference after incubation with $1 \mathrm{mg} / \mathrm{mL}$ Suxiao Jiuxin Pill compared to the control $(P=0.025$; 95\% CI: $-1.9,16.2 \mathrm{mN})$. Pretreatment of IMA with Suxiao Jiuxin Pill $(10 \mathrm{mg} / \mathrm{mL})$ abolished the subsequent contraction to $\mathrm{KCl}$ (Figure 2(a)). Figures 2(b) and 2(c) reproduced our previous data on the relaxing effect of the well-studied western vasorelaxant agents glyceryl trinitrate (Figure 2(b)) and amlodipine (Figure 2(c)) at the plasma concentration in comparison to Suxiao Jiuxin Pill. In comparison to glyceryl trinitrate and amlodipine, the 


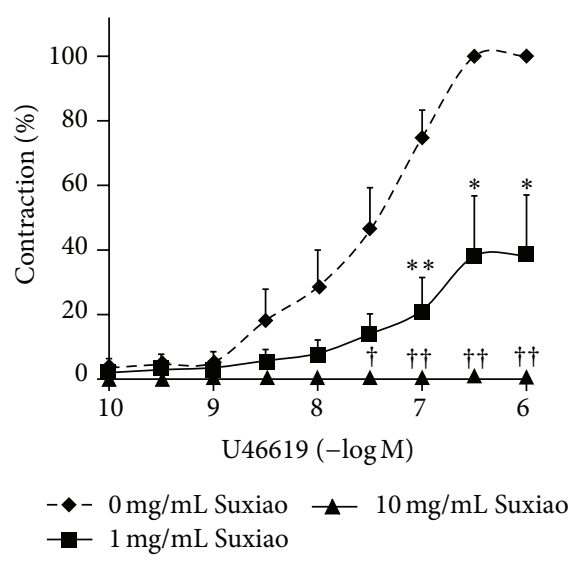

(a)

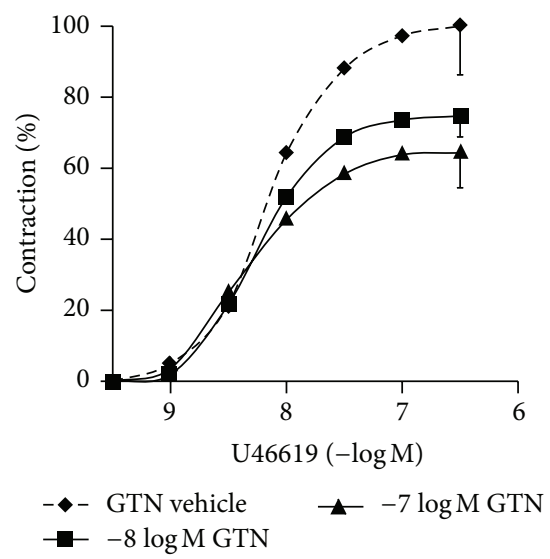

(b)

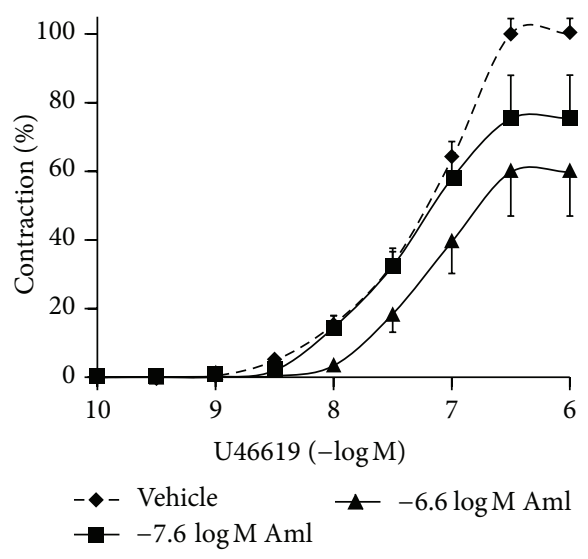

(c)

Figure 3: Mean concentration- $(-\log M)$ contraction $(\mathrm{mN})$ curves for U46619 when Suxiao Jiuxin Pill $1 \mathrm{mg} / \mathrm{mL}(\boldsymbol{\square}), 10 \mathrm{mg} / \mathrm{mL}(\mathbf{\Delta})$, or vehicle

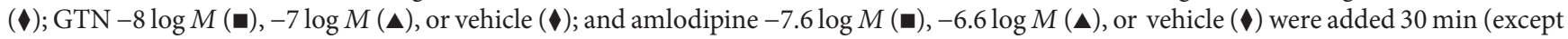
for GTN were added $10 \mathrm{~min}$ ) before the contraction started in the human IMA ring segments ( $n=6$ in each group). Three rings from an IMA segment taken from the same patient were allocated into each group. Values are expressed as mean \pm SEM. ${ }^{*} P<0.05$ compared to vehicle group in $1 \mathrm{mg} / \mathrm{mL}$ Suxiao Jiuxin Pill pretreatment; ${ }^{\dagger} P<0.05$ compared to vehicle group in $10 \mathrm{mg} / \mathrm{mL}$ Suxiao Jiuxin Pill pretreatment (unpaired $t$-test). KCl: potassium chloride; GTN: glyceryl trinitrate; Suxiao: Suxiao Jiuxin Pill; Aml: amlodipine.

magnitude of the maximal contraction to $\mathrm{KCl}$ was significantly depressed by the plasma concentration of Suxiao Jiuxin Pill $(1 \mathrm{mg} / \mathrm{mL})$ as mentioned above (Figure $2(\mathrm{a}))$ but it had no significant change in the plasma concentration of glyceryl trinitrate- $(-8 \log M)$ pretreated (Figure $2(\mathrm{~b})$ ) or amlodipine$(-7.6 \log M)$ pretreated (Figure 2(c)) IMA, although higher concentration of amlodipine $(-6.6 \log M)$ also significantly depressed the maximal contraction of IMA (Figure 2(c)).

Further, in IMA rings contracted by U46619, incubation with $1 \mathrm{mg} / \mathrm{mL}$ Suxiao Jiuxin Pill significantly depressed the magnitude of the maximal contraction of U46619 $(P=$ 0.007 ; $95 \%$ CI: $4.9 ; 22.6 \mathrm{mN}$; unpaired $t$-test). Two-way ANOVA for all concentrations of U46619 demonstrated no significant differences compared to the control $(P=0.058$; $95 \% \mathrm{CI}:-0.8 ; 5.1 \mathrm{mN})$. In addition, pretreatment of IMA with Suxiao Jiuxin Pill (10 mg/mL) abolished the subsequent contraction to U46619 (Figure 3). In comparison to the effect of pretreatment with glyceryl trinitrate (Figure 3(b)) and amlodipine (Figure 3(c)), neither the plasma concentration ( $-8 \log M$ for glyceryl trinitrate and $-7.6 \log M$ for amlodipine) nor even the 10 -fold higher concentrations significantly reduced the maximal contraction to U46619. These data were standardized with percentage of the maximal contraction for easier comparison.

These results show that in comparison to the effect of pretreatment with these three vasorelaxant agents at the plasma concentration, only Suxiao Jiuxin Pill caused significant depression effect of the contraction to either $\mathrm{KCl}$ or U46619.

3.4. At the Concentrations of Less Than $1 \mathrm{mg} / \mathrm{mL}$, Suxiao Jiuxin Pill-Induced Relaxation Is Altered by Endothelium-Denudation and L-NNA. At the concentrations of $31.6 \mu \mathrm{g} / \mathrm{mL}$,
$100 \mu \mathrm{g} / \mathrm{mL}$, and $316 \mu \mathrm{g} / \mathrm{mL}$, Suxiao Jiuxin Pill-induced relaxation against $40 \mathrm{mM} \mathrm{KCl}$ was significantly attenuated by denudation of endothelium $(0.3 \pm 0.3 \%$ versus $4.6 \pm 1.8 \%$, $P<0.05$ for $31.6 \mu \mathrm{g} / \mathrm{mL}$ Suxiao Jiuxin Pill; $0.7 \pm 0.5 \%$ versus $9.3 \pm 3.4 \%, P<0.05$ for $100 \mu \mathrm{g} / \mathrm{mL}$ Suxiao Jiuxin Pill; $5.4 \pm$ $2.1 \%$ versus $24.9 \pm 5.6 \%, P<0.01$ for $316 \mu \mathrm{g} / \mathrm{mL}$ Suxiao Jiuxin Pill; Figure 4(a)). A similar effect was seen in endotheliumintact IMA rings pretreated with $300 \mu \mathrm{M}$ L-NNA $(0.4 \pm 0.3 \%$ versus $4.6 \pm 1.8 \%, P<0.05$ for $31.6 \mu \mathrm{g} / \mathrm{mL}$ Suxiao Jiuxin Pill; $1.3 \pm 0.7 \%$ versus $9.3 \pm 3.4 \%, P<0.05$ for $100 \mu \mathrm{g} / \mathrm{mL}$ Suxiao Jiuxin Pill; $8.1 \pm 2.4 \%$ versus $24.9 \pm 5.6 \%, P<0.05$ for $316 \mu \mathrm{g} / \mathrm{mL}$ Suxiao Jiuxin Pill; Figure 4(b)).

Taken together, Figure 4 shows that the effect of denudation of the endothelium and addition of NO-inhibitor LNNA are similar. At the concentrations of less than $1 \mathrm{mg} / \mathrm{mL}$, Suxiao Jiuxin Pill-induced relaxation is reduced by both endothelium-denudation and L-NNA. However, the maximal relaxation was not altered. These results show that Suxiao Jiuxin Pill-induced relaxation is mainly endotheliumindependent although it also has a small endothelium-dependent component.

3.5. Western Blot Analysis of eNOS in IMA Pretreated with Suxiao Jiuxin Pill. The eNOS expressions in IMA had no significant differences between the control and Suxiao Jiuxin Pill (1 mg/mL or $10 \mathrm{mg} / \mathrm{mL}$ ) pretreated groups (Figure 5).

\section{Discussion}

In this study we have found in the human IMA, the most commonly used arterial graft for coronary artery bypass surgery, that (1) the compound Chinese medicine Suxiao Jiuxin Pill 


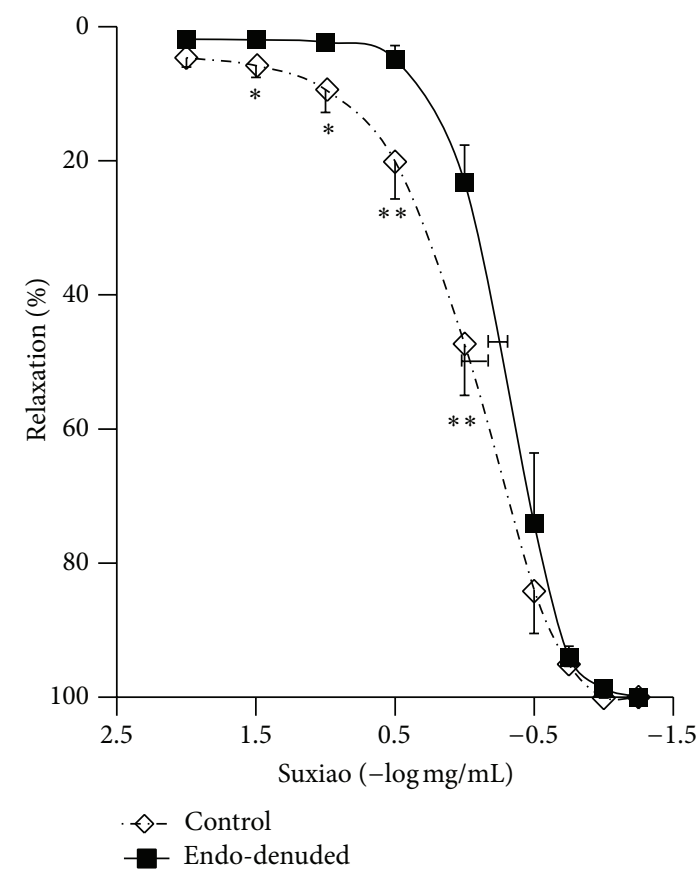

(a)

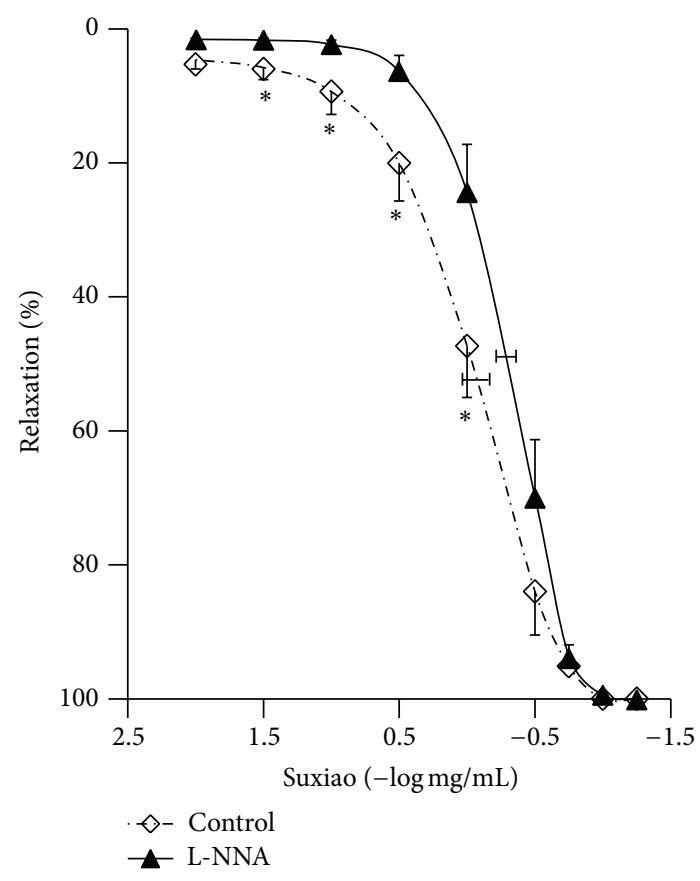

(b)

Figure 4: Mean concentration- (log mg/mL) relaxation (\%) curves for Suxiao Jiuxin Pill (-2 1.25 log mg/mL) in endothelium-denuded $(n=6$; (a) ) and L-NNA $(300 \mu \mathrm{M}, n=6$; (b)) pretreated IMA rings precontracted with $40 \mathrm{mM} \mathrm{KCl}$. Endothelium-intact IMA rings taken from the same patient were studied as control. Values are expressed as mean \pm SEM. ${ }^{*} P<0.05,{ }^{* *} P<0.01$, compared with endotheliumintact control group. Suxiao: Suxiao Jiuxin Pill; Endo-denuded: endothelium-denuded.

had potent vasorelaxant effect on various vasoconstrictormediated vasoconstriction, particularly on $\mathrm{KCl}$ and U46619; (2) Suxiao Jiuxin Pill fully relaxed the contraction caused by $\mathrm{KCl}$ and U46619 with similar potencies; (3) pretreatment with Suxiao Jiuxin Pill effectively prevented and abolished $\mathrm{KCl}$ - or U46619-induced contraction and this effect may be more significant than the effect caused by the pretreatment by GTN and amlodipine; (4) the mechanism of Suxiao Jiuxin Pill relaxing human arteries may involve endothelium-dependent mechanism at the concentrations of microgram per milliliter; and (5) Suxiao Jiuxin Pill at concentrations of milligram per milliliter presents endothelium-independent relaxation.

With more than 2 decades of clinical application, large amount of clinical pharmacological studies has proved that Suxiao Jiuxin Pill has anti-atherosclerosis effect, may improve microenvironment, and may protect myocytes and vascular endothelial cells [47]. Moreover, long-term Suxiao Jiuxin Pill intake may also significantly improve heart function and decrease angina pectoris attack as well as protect patients from myocardial infarction [47]. These effects have been obtained not only for emergency lifesaving but also for routine therapy. It is also reported that with the special advantage of no drug tolerance and toxic or side effect, it greatly supplements the deficiency of nitrate esters for daily taking [47]. Owing to the above-mentioned advantages, Suxiao Jiuxin Pill is now widely used in the treatment of coronary artery disease.

As mentioned above, the main components of Suxiao Jiuxin Pill are tetramethylpyrazine (TMP) and borneol, although there are also a number of other substances contained in this medicine. In recent years, a variety of studies have been performed to investigate the therapeutic effect of TMP, including the vasodilatation and endothelial protection. A study shows that TMP is a dilator of human pulmonary and bronchial arteries through endotheliumindependent mechanism and that TMP preferentially relaxes pulmonary resistance vessels rather than large conduit pulmonary arteries [48]. It has also been shown that TMP elicits disparate responses in cardiac contraction and intracellular $\mathrm{Ca}(2+)$ transients in isolated adult rat ventricular myocytes [12]. TMP in treating human arteriosclerosis obliterans not only displays extraordinary effect, but also has good effect on curing the damage of endothelial cells [49]. Moreover, TMP has been shown to have protective effects on the vascular endothelial function in patients undergoing PCI [50] and on $\mathrm{H}_{2} \mathrm{O}_{2}$-induced oxidative damage in human umbilical vein endothelial cells due to its antioxidant and antiapoptotic properties by downregulating nitric oxide (NO) and nitric oxide synthase (NOS) production [49] and by scavenging ROS and regulating intracellular calcium concentration [51]. In addition, TMP at micromolar concentrations stimulated NO production in human platelets via a novel mechanism that activated eNOS protein expression [52] and produced a concentration-dependent relaxation in the aortic rings precontracted with vasopressin or phenylephrine that is related to the opening of $\mathrm{SK}_{\mathrm{Ca}}$ and $\mathrm{K}_{\mathrm{ATP}}$ channels [53].

In comparison to the well-studied component TMP, the role of borneol in Suxiao Jiuxin Pill is less clear but 

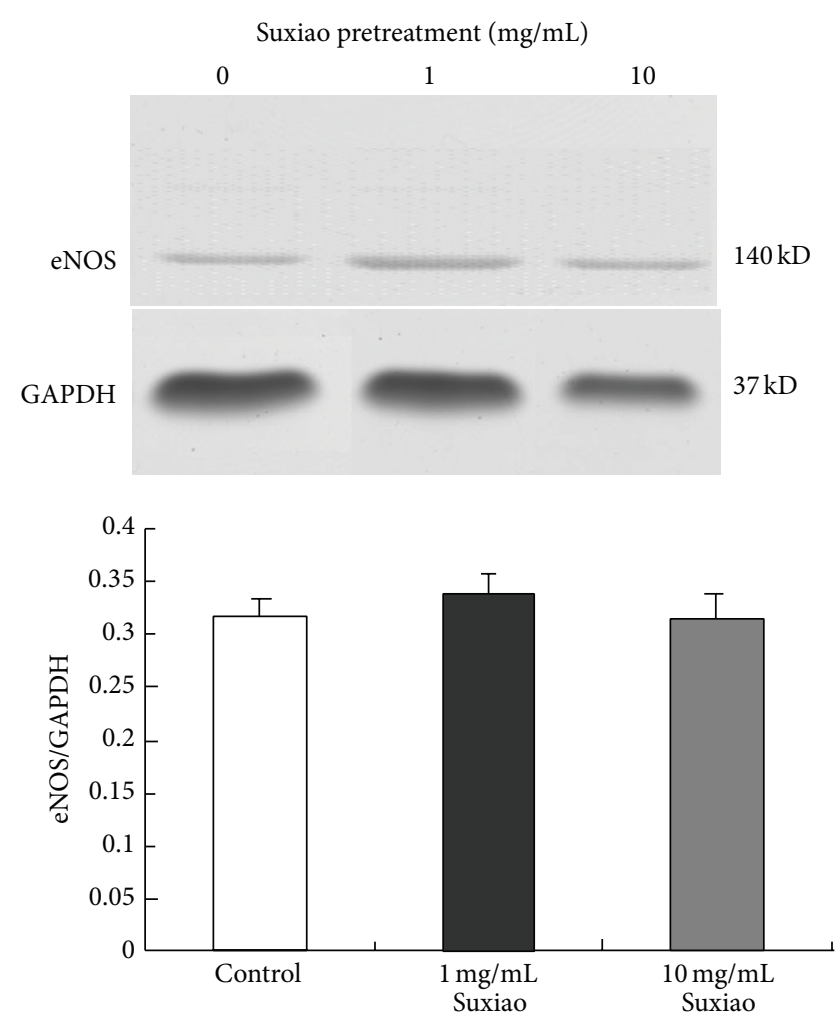

FIGURE 5: Protein expression of eNOS in human IMA rings. IMA rings were incubated with Suxiao Jiuxin Pill $1 \mathrm{mg} / \mathrm{mL}, 10 \mathrm{mg} / \mathrm{mL}$, or vehicle (Control) for $30 \mathrm{~min}(n=3)$. Data are shown as mean $\pm \mathrm{SEM}$. Suxiao: Suxiao Jiuxin Pill; eNOS: endothelial nitric oxide synthase.

this component is frequently used in traditional Chinese medicine [54]. There is lack of studies on the effect of borneol on vascular tone. However, borneol may have benefits for the neuroprotective effect of sodium ferulate against injury induced in the brain by ischaemia/reperfusion [55]. More likely, borneol may promote the absorption of other components in the compound Chinese medicine as it has been shown that borneol promoted nasal absorption of Ligustrazine into brain [56].

On the basis of the above findings, we explored the vasorelaxation of Suxiao Jiuxin Pill and possible mechanisms in the human IMA in the present study. We demonstrated that Suxiao Jiuxin Pill relaxed all IMA segments against various vasoconstrictors representing different vasoconstriction mechanisms, including $\mathrm{KCl}$ and $\mathrm{U} 46619 . \mathrm{KCl}$ was used to depolarize the smooth muscle cells to open the voltageoperated channel (VOC) [57], whereas U46619 may bind the receptors to activate the receptor-operated channel (ROC) [29]. The results show that Suxiao Jiuxin Pill has full relaxant effects on contractions induced by both vasoconstrictors with similar potencies, indicating a high effectiveness of this vasodilator agent (Figure 1(a)). In comparison, in our previous study, the calcium antagonist amlodipine induced full relaxation in contractions mediated by these two vasoconstrictors but the potency was higher in the vasoconstriction mediated by $\mathrm{KCl}$ than that mediated by $\mathrm{U} 46619$
[43]. In contrast, Suxiao Jiuxin Pill rapidly relaxed the IMA regardless of the nature of the constrictor stimulus. Further, we previously demonstrated that GTN could fully relax the IMA precontracted by $\mathrm{KCl}$ or U46619 with similar $\mathrm{EC}_{50}$ values [29]. On this point, Suxiao Jiuxin Pill is similar to GTN with no significant constrictor selectivity in the relaxation.

Importantly, Suxiao Jiuxin Pill had significant effect on inhibition if used prior to contraction. Indeed, pretreatment of Suxiao Jiuxin Pill at $1 \mathrm{mg} / \mathrm{mL}$ significantly depressed the contraction mediated by both $\mathrm{KCl}$ and U46619 and at $10 \mathrm{mg} / \mathrm{mL}$, the contraction was even abolished (Figures 2(a) and 3(a)). This effect was significantly higher than that by the plasma concentration of either GTN or amlodipine (compare Figures 2(a)-2(c) and 3(a)-3(c)). Amlodipine is known as a calcium antagonist [58]. The clinical implication of this result is strong. This implies that at the plasma concentration, the antispastic effect (prevention of strong contraction) of Suxiao Jiuxin Pill is significantly higher than that of either GTN or amlodipine at the plasma concentration of these vasodilators. Such effect may be favorable for the patients who have angina pectoris or the patients who have had CABG operation with IMA grafting for prophylactic therapy of vasospasm. Our findings are in accordance with the recent report demonstrating that Suxiao Jiuxin Pill could improve the pre- and post-PCI coronary artery flow rate, increase the collateral artery patency, and reduce the incidence of perioperative myocardial infarction of acute coronary syndrome patients [59].

The present study clearly shows that Suxiao Jiuxin Pill -induced relaxation in the human artery has two components-endothelium-dependent and -independent mechanisms. The endothelium-dependent mechanism is the major component of the relaxation; the endothelium-dependent effect exists but it is a small effect. At microgram per milliliter concentrations the relaxation is endothelium-dependent, as shown in Figure 4. Indeed, either endothelium-denudation or L-NNA inhibited the Suxiao Jiuxin Pill-induced relaxation at the lower concentrations. At higher concentrations (higher than $1 \mathrm{mg} / \mathrm{mL}$ ), however, the role of endothelium became insignificant. The endothelium-independent relaxation was obviously due to the direct relaxing effect of Suxiao Jiuxin Pill on the smooth muscle. Interestingly, western blot analysis showed that Suxiao Jiuxin Pill had no effect on the protein expression of eNOS (Figure 5). Taken together with the result that the eNOS inhibitor L-NNA had inhibitory effect on the relaxation, the present study suggests that the endotheliumdependent component at lower concentrations of Suxiao Jiuxin Pill is most likely associated with posttranslational modification of eNOS, which, however, warrants further investigation.

In conclusion, the present study suggests that Suxiao Jiuxin Pill has a rapid and potent vasorelaxant effect on human IMA precontracted by a variety of vasoconstrictors with similar potency. Pretreatment with Suxiao Jiuxin Pill has high potency in inhibiting contraction induced by membrane depolarization and receptor (thromboxane) mechanisms. The vasorelaxation induced by Suxiao Jiuxin Pill involves both endothelium-dependent and -independent 
mechanisms. The present study demonstrates a full and effective vasorelaxation effect of Suxiao Jiuxin Pill and supports the use of this widely used medication in coronary artery disease including CABG patients, in favor of treating and preventing graft spasm. Therefore, the effect of Suxiao Jiuxin Pill on human arteries demonstrated in this study may prove to be particularly important in vasorelaxing therapy in cardiovascular disease.

\section{Conflict of Interests}

The authors disclose that this research was partially supported by Tianjin Zhongxin Pharmaceutical Group Co., Ltd. Tianjin, China.

\section{Acknowledgments}

The work described in this paper was fully supported by Grants from the National Natural Science Foundation of China (no. 81170148), the National Basic Research Program of China (no. 2010CB529500), International S \& T Cooperation Program of China (no. 2009DFB30560),National Science and Technology Major Project (2013ZX09303004-005), Tianjin Municipal Science and Technology Commission, 09ZCZDSF04200 and 10JCYBJC26400, Tianjin Municipal Research Grant for Applied Basic and Frontier Technology, China, Binhai Key Platform for Creative Research Program (2012-BH110004), Tianjin Binhai New Area Health Bureau (2011BHKZ001, 2012BWKZ 008, 2011BHKY002, and 2012 BWKY024), Tianjin Health Bureau (2012KZ009, 2012KZ010, and 2013KZ009), and Hong Kong Research Grants Council GRF Grants (CUHK4774/12). The assistance of the surgical team and nurses (Yu En-Jie and the team members) in the Cardiac Operating Theater, TEDA International Cardiovascular Hospital, is gratefully acknowledged.

\section{References}

[1] M. L. Daviglus, D. M. Lloyd-Jones, and A. Pirzada, "Preventing cardiovascular disease in the 21st century: therapeutic and preventive implications of current evidence," The American Journal of Cardiovascular Drugs, vol. 6, no. 2, pp. 87-101, 2006.

[2] E. G. Nabel and E. Braunwald, "A tale of coronary artery dis-ease and myocardial infarction," The New England Journal of Medicine, vol. 366, no. 1, pp. 54-63, 2012.

[3] S. R. Sun, X. Huang, and L. Zhang, "Advances in studies on pharmacokinetics, pharmacodynamics and clinical research of Suxiao Jiuxin Wan," Chinese Traditional and Herbal Drugs, vol. 33, pp. 89-91, 2002.

[4] X. Duan, L. Zhou, T. Wu et al., "Chinese herbal medicine Suxiao Jiuxin wan for angina pectoris," Cochrane Database of Systematic Reviews, no. 1, Article ID CD004473, 2008.

[5] L. Feng, T. Han, and Y. L. Zhou, “. The conclusion of the clinic effect for Suxiao Jiuxin Wan for CHD and angina pectoris -," Journal of Emergency Syndromes in Chinese Medicine, vol. 9, pp. 4-6, 2000.

[6] C. S. Li, Z. Q. Qu, S. S. Wang et al., "Effects of Suxiao Jiuxin Pill on oxidative stress and inflammatory response in rats with experimental atherosclerosis," Journal of Traditional Chinese Medicine, vol. 31, no. 2, pp. 107-111, 2011.
[7] G. Yuan, "Curative effect observions on Suxiao Jiuxin Wan compared to Xiao xin tong for Angina pectoris," Medical Journal of Healing of Heart and Vessel, vol. 9, pp. 71-72, 2002.

[8] J. Wei and Y. Tang, "Comparing research on the clinical effect of Suxiao Jiuxin Wan for ischemic heart disease," Journal of Emergency Syndromes in Chinese Medicine, vol. 164, pp. 159-160, 1995.

[9] L. L. Yang, Y. S. Yuan, Y. L. Tong, and C. Y. Guo, "Determination of tetramethylpyrazine and borneol in human plasma by GCMSD," Yao Xue Xue Bao, vol. 29, no. 9, pp. 697-701, 1994 (Chinese).

[10] S. F. Dong and Z. G. Zhu, "Determination of the Contents of $\mathrm{Ca}, \mathrm{Mg}, \mathrm{Fe}, \mathrm{Cu}$ and $\mathrm{Zn}$ in Suxiao Jiuxin Pill and the Analysis of $\mathrm{Ca} / \mathrm{Mg}$ and $\mathrm{Cu} / \mathrm{Zn}$ Values," Guang Pu Xue Yu Guang Pu Fen Xi, vol. 22, no. 3, pp. x23-479, 2002 (Chinese).

[11] State Administration of Traditional Chinese Medicine (SATCM) of the P.R. of China: Zhong Hua Ben Cao, Volume V:15, pp. 976-984. Shanghai Science \& Technology Press, 2007.

[12] K. K. Hintz and J. Ren, "Tetramethylpyrazine elicits disparate responses in cardiac contraction and intracellular $\mathrm{Ca}^{2+}$ transients in isolated adult rat ventricular myocytes," Vascular Pharmacology, vol. 40, no. 4, pp. 213-217, 2003.

[13] L. Feng, Y. Xiong, F. Cheng, L. Zhang, S. Li, and Y. Li, "Effect of ligustrazine on ischemia-reperfusion injury in murine kidney," Transplantation Proceedings, vol. 36, no. 7, pp. 1949-1951, 2004.

[14] L. B. Esberg and J. Ren, "The oxygen radical generator pyrogallol impairs cardiomyocyte contractile function via a superoxide and p38 MAP kinase-dependent pathway: protection by anisodamine, tetramethylpyrazine," Cardiovascular Toxicology, vol. 4, no. 4, pp. 375-384, 2004.

[15] Z. Zhang, T. Wei, J. Hou, G. Li, S. Yu, and W. Xin, "Iron-induced oxidative damage and apoptosis in cerebellar granule cells: attenuation by tetramethylpyrazine and ferulic acid," European Journal of Pharmacology, vol. 467, no. 1-3, pp. 41-47, 2003.

[16] Y. S. Fu, Y. Y. Lin, S. C. Chou et al., "Tetramethylpyrazine inhibits activities of glioma cells and glutamate neuro-excitotoxicity: potential therapeutic application for treatment of gliomas," Neuro-Oncology, vol. 10, no. 2, pp. 139-152, 2008.

[17] Y. H. Shih, S. L. Wu, W. F. Chiou, H. H. Ku, T. L. Ko, and Y. S. Fu, "Protective effects of tetramethylpyrazine on kainate induced excitotoxicity in hippocampal culture," Neuroreport, vol. 13, no. 4, pp. 515-519, 2002.

[18] S. L. Liao, T. K. Kao, W. Y. Chen et al., "Tetramethylpyrazine reduces ischemic brain injury in rats," Neuroscience Letters, vol. 372, no. 1-2, pp. 40-45, 2004.

[19] K. C. Wong, K. S. Ong, and C. L. Lim, "Composition of the Essential Oil of Rhizomes of Kaempferia galanga L," Flavour and Fragrance Journal, vol. 7, no. 5, pp. 263-266, 1992.

[20] X. W. Li, J. Li, and H. der Werff, 'Cinnamomum camphora'. Flora of China. Missouri Botanical Garden, St. Louis, Mo, USA \& Harvard University Herbaria, Cambridge, Mass, USA, 2013.

[21] H. Song, T. Guo, R. Zhang et al., "Preparation of the traditional Chinese medicine compound recipe heart-protecting musk pH-dependent gradient-release pellets," Drug Development and Industrial Pharmacy, vol. 28, no. 10, pp. 1261-1273, 2002.

[22] G. W. He and C. Q. Yang, "Comparison among arterial grafts and coronary artery: an attempt at functional classification," Journal of Thoracic and Cardiovascular Surgery, vol. 109, no. 4, pp. 707-715, 1995.

[23] G. W. He, "Arterial grafts for coronary artery bypass grafting: biological characteristics, functional classification, and clinical choice," Annals of Thoracic Surgery, vol. 67, no. 1, pp. 277-284, 1999. 
[24] B. W. Lytle, E. H. Blackstone, J. F. Sabik, P. Houghtaling, F. D. Loop, and D. M. Cosgrove, "The effect of bilateral internal thoracic artery grafting on survival during 20 postoperative years," Annals of Thoracic Surgery, vol. 78, no. 6, pp. 2005-2014, 2004.

[25] D. P. Taggart, R. D'Amico, and D. G. Altman, "Effect of arterial revascularisation on survival: a systematic review of studies comparing bilateral and single internal mammary arteries," The Lancet, vol. 358, no. 9285, pp. 870-875, 2001.

[26] M. C. Sarabu, J. A. McClung, A. Fass, and G. E. Reed, "Early postoperative spasm in left internal mammary artery bypass grafts," Annals of Thoracic Surgery, vol. 44, no. 2, pp. 199-200, 1987.

[27] P. R. Vogt, O. Hess, and M. I. Turina, "Internal mammary artery spasm immediately after grafting to the left anterior descending artery: diagnosis and treatment," European Heart Journal, vol. 17, no. 5, p. 804, 1996.

[28] G. W. Stone and G. O. Hartzler, "Spontaneous reversible spasm in an internal mammary artery graft causing acute myocardial infarction," The American Journal of Cardiology, vol. 64, no. 12, pp. 822-823, 1989.

[29] G. W. He, F. L. Rosenfeldt, B. F. Buxton, and J. A. Angus, "Reactivity of human isolated internal mammary artery to constrictor and dilator agents: implications for treatment of internal mammary artery spasm," Circulation, vol. 80, no. 3, pp. I141-I150, 1989.

[30] H. Tatar, S. Cicek, H. Suer, A. T. Yilmaz, O. Y. Ozturk, and R. D. Narbay, "Use of topical sodium nitroprusside to relieve perioperative spasm of the internal mammary artery: a word of caution," Journal of Thoracic and Cardiovascular Surgery, vol. 108, no. 2, p. 387, 1994.

[31] D. Zabeeda, B. Medalion, S. Jackobshvilli, S. Ezra, A. Schachner, and A. J. Cohen, "Comparison of systemic vasodilators: effects on flow in internal mammary and radial arteries," Annals of Thoracic Surgery, vol. 71, no. 1, pp. 138-141, 2001.

[32] A. Novaković, L. Gojković-Bukarica, B. Beleslin-Cokić et al., "Differential antivasoconstrictor effects of levcromakalim and rilmakalim on the isolated human mammary artery and saphenous vein," Journal of Pharmacological Sciences, vol. 92, pp. 108114, 2003.

[33] L. H. Sanders and M. A. Newman, "Intractable postoperative internal thoracic artery spasm managed with angiographic intraluminal papaverine," Journal of Thoracic and Cardiovascular Surgery, vol. 130, no. 3, pp. 938-940, 2005.

[34] R. E. Harskamp, J. D. McNeil, M. W. van Ginkel, R. B. Bastos, C. E. Baisden, and J. H. Calhoon, "Postoperative internal thoracic artery spasm after coronary artery bypass grafting," Annals of Thoracic Surgery, vol. 85, no. 2, pp. 647-649, 2008.

[35] G. W. He, "Verapamil plus nitroglycerin solution maximally preserves endothelial function of the radial artery: comparison with papaverine solution," Journal of Thoracic and Cardiovascular Surgery, vol. 115, no. 6, pp. 1321-1327, 1998.

[36] Z. W. Chen, Y. Huang, Q. Yang, X. Li, W. Wei, and G. W. He, "Urocortin-induced relaxation in the human internal mammary artery," Cardiovascular Research, vol. 65, no. 4, pp. 913920, 2005.

[37] Z. W. Chen, Q. Yang, Y. Huang, L. Fan, X. W. Li, and G. W. He, "Human urotensin II in internal mammary and radial arteries of patients undergoing coronary surgery," Vascular Pharmacology, vol. 52, no. 1-2, pp. 70-76, 2010.
[38] G. W. He and Z. G. Liu, "Comparison of nitric oxide release and endothelium-derived hyperpolarizing factor-mediated hyperpolarization between human radial and internal mammary arteries," Circulation, vol. 104, no. 1, pp. I344-I349, 2001.

[39] G. W. He, J. A. Angus, and F. L. Rosenfeldt, "Reactivity of the canine isolated internal mammary artery, saphenous vein, and coronary artery to constrictor and dilator substances: Relevance to coronary bypass graft surgery," Journal of Cardiovascular Pharmacology, vol. 12, no. 1, pp. 12-22, 1988.

[40] X. Y. Bai, X. C. Liu, Q. Yang, X. D. Tang, and G. W. He, “The interaction between human urotensin II and vasodilator agents in human internal mammary artery with possible clinical implications," Annals of Thoracic Surgery, vol. 92, no. 2, pp. 610616, 2011.

[41] H. Wu, J. Guo, X. H. Li, and X. Huang, "Determination of Borneol in Blood After Keeping Suxiao Jiuxin Pills in Healthy People's Mouth by GC," China Pharmacy, vol. 14, pp. 414-415, 2003.

[42] J. Guo, X. Huang, L. L. Wang, H. Meng, L. Zhang, and P. Ren, "Synchro-determ ination of borneol and tetramethylpyrazine in plasma by GC-FID for volunteers administrated with Suxiao Jiuxin Wan," Chinese Traditional and Herbal Drugs, vol. 34, pp. 730-732, 2003.

[43] X. Y. Bai, X. C. Liu, W. B. Jing, Q. Yang, X. D. Tang, and G. W. $\mathrm{He}$, "Effect of amlodipine in human internal mammary artery and clinical implications," Annals of Thoracic Surgery, vol. 90, no. 6, pp. 1952-1957, 2010.

[44] G. Gao, X. Y. Bai, C. Xuan et al., "Role of TRPC3 channel in human internal mammary artery," Archives of Medical Research, vol. 43, pp. 431-437, 2012.

[45] H. M. Xue, G. W. He, J. H. Huang, and Q. Yang, "New strategy of endothelial protection in cardiac surgery: Use of enhancer of endothelial nitric oxide synthase," World Journal of Surgery, vol. 34, no. 7, pp. 1461-1469, 2010.

[46] Q. Yang, H. M. Xue, W. T. Wong et al., "AVE3085, an enhancer of endothelial nitric oxide synthase, restores endothelial function and reduces blood pressure in spontaneously hypertensive rats," British Journal of Pharmacology, vol. 163, no. 5, pp. 1078-1085, 2011.

[47] S. Wang, "The Study on Pharmacological and Clinical Research of Suxiao Jiuxin Wan," Zhongguo Lin Chuang Yi Yao Yan Jiu Za Zhi, vol. 178, pp. 42-45, 2007.

[48] S. F. Liu, Y. N. Cai, T. W. Evans, D. G. McCormack, G. R. Barer, and P. J. Barnes, "Ligustrazine is a vasodilator of hu-man pulmonary and bronchial arteries," European Journal of Pharmacology, vol. 191, no. 3, pp. 345-350, 1990.

[49] S. Li, P. Huang, and C. Liu, "Effect of ligustrazine injection on vascular endothelial cell of the patients with arteriosclerosis obliterans," Zhongguo Zhong Xi Yi Jie He Za Zhi, vol. 20, no. 6, pp. 412-414, 2000.

[50] Z. Q. Chen, L. Hong, and H. Wang, "Effect of tetramethylpyrazine on platelet activation and vascular endothelial function in patients with acute coronary syndrome undergoing percutaneous coronary intervention," Zhongguo Zhong Xi Yi Jie He Za Zhi, vol. 27, no. 12, pp. 1078-1081, 2007.

[51] Y. Ou, X. L. Guo, L. Zhai, X. Y. Liu, and Y. N. Cheng, “TMPDP, a tetramethylpyrazine derivative, protects vascular endothelial cells from oxidation damage by hydrogen peroxide," Pharmazie, vol. 65, no. 10, pp. 755-759, 2010.

[52] J. R. Sheu, Y. C. Kan, W. C. Hung, C. H. Lin, and M. H. Yen, "The antiplatelet activity of tetramethylpyrazine is mediated through 
activation of NO synthase," Life Sciences, vol. 67, no. 8, pp. 937947, 2000.

[53] C. C. Tsai, T. Y. Lai, W. C. Huang, I. M. Liu, and J. T. Cheng, "Inhibitory effects of potassium channel blockers on tetramethylpyrazine-induced relaxation of rat aortic strip in vitro," Life Sciences, vol. 71, no. 11, pp. 1321-1330, 2002.

[54] X. Li, L. Wu, X. Fan et al., "Network pharmacology study on major active compounds of Fufang Danshen formula," Zhongguo Zhongyao Zazhi, vol. 36, no. 21, pp. 2911-2915, 2011.

[55] X. H. Chen, Z. Z. Lin, A. M. Liu et al., "The orally combined neuroprotective effects of sodium ferulate and borneol against transient global ischaemia in C57 BL/6J mice," Journal of Pharmacy and Pharmacology, vol. 62, no. 7, pp. 915-923, 2010.

[56] Y. D. Liu, H. J. Sun, R. Li, W. Wu, and H. G. Chen, "Influence of borneol on nasal absorption of Ligustrazinee," Zhongguo Zhongyao Zazhi, vol. 33, no. 3, pp. 259-261, 2008.

[57] T. B. Bolton, "Mechanisms of action of transmitters and other substances on smooth muscle," Physiological Reviews, vol. 59, no. 3, pp. 606-718, 1979.

[58] S. B. Kribbs, W. M. Merritt, M. J. Clair et al., "Amlodipine monotherapy, angiotensin-converting enzyme inhibition, and combination therapy with pacing-induced heart failure," Hypertension, vol. 31, no. 3, pp. 755-765, 1998.

[59] X. L. Wang, Y. M. Liu, and G. J. Zhu, "Effects of Suxiao Jiuxin pill on patients with acute coronary syndrome undergoing early percutaneous coronary intervention," Zhongguo Zhong Xi Yi Jie He Za Zhi, vol. 32, pp. 1483-1487, 2012 (Chinese). 


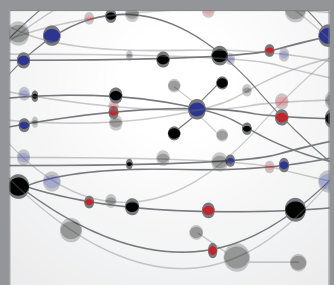

The Scientific World Journal
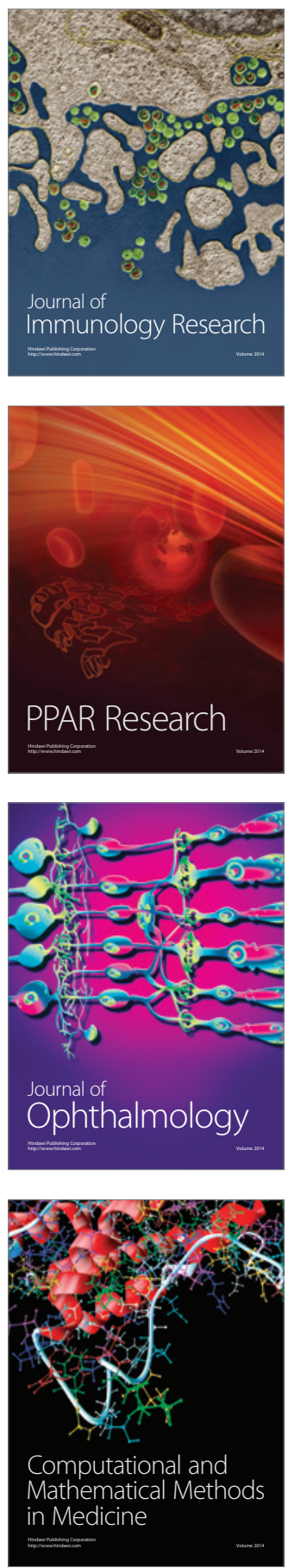

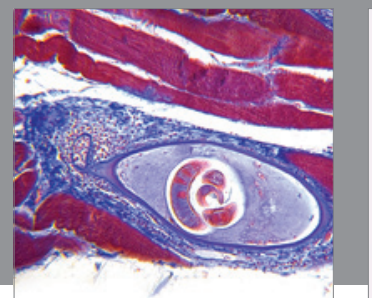

Gastroenterology

Research and Practice
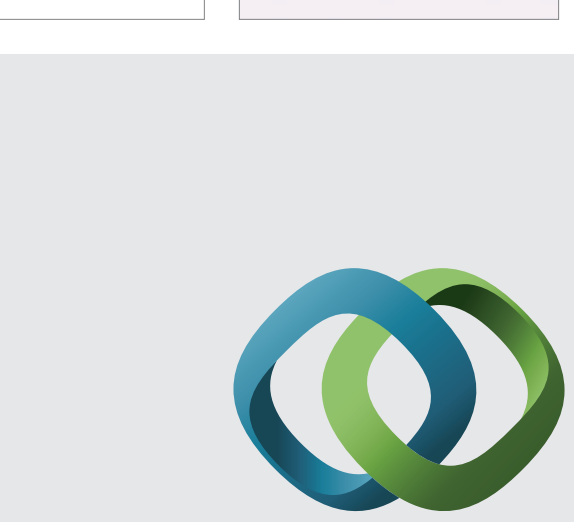

\section{Hindawi}

Submit your manuscripts at

http://www.hindawi.com
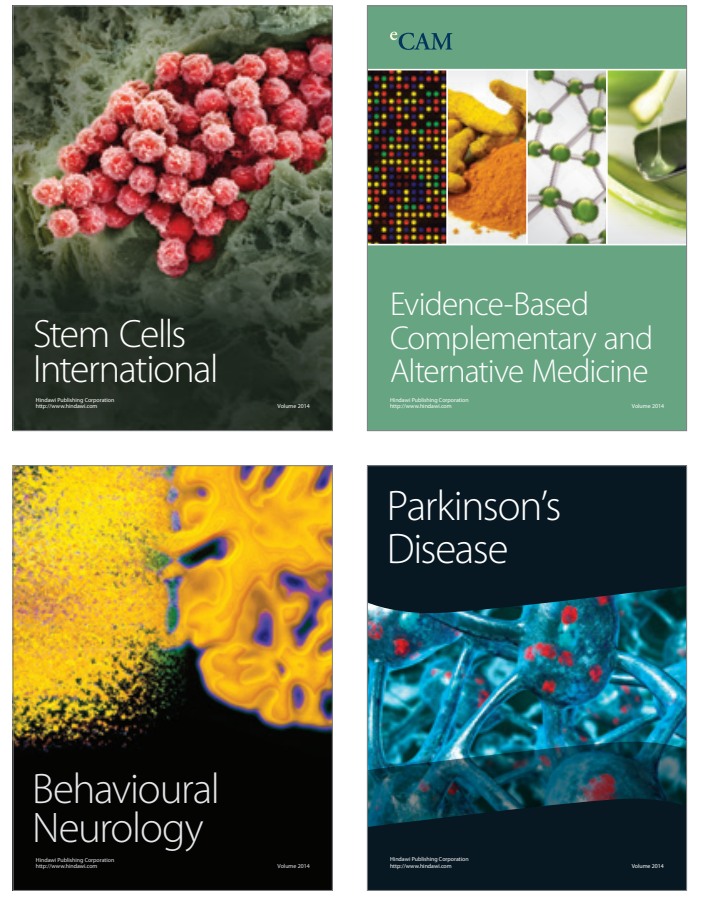
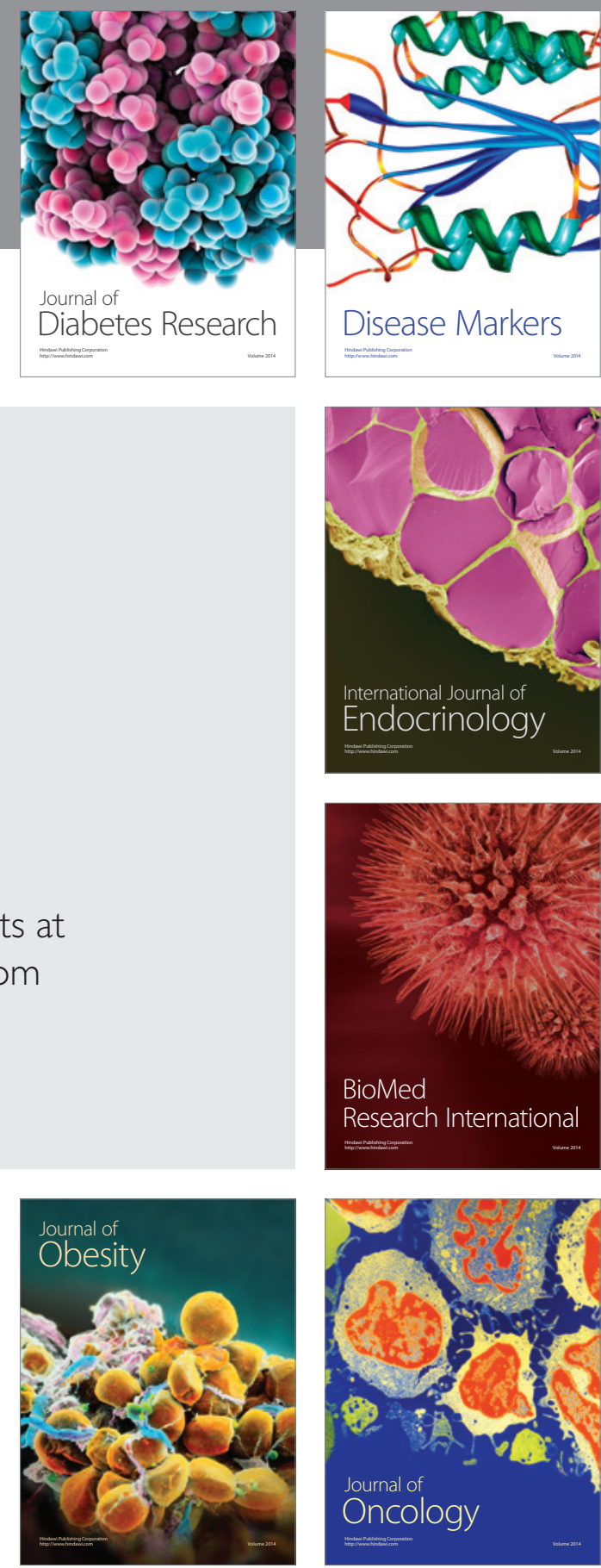

Disease Markers
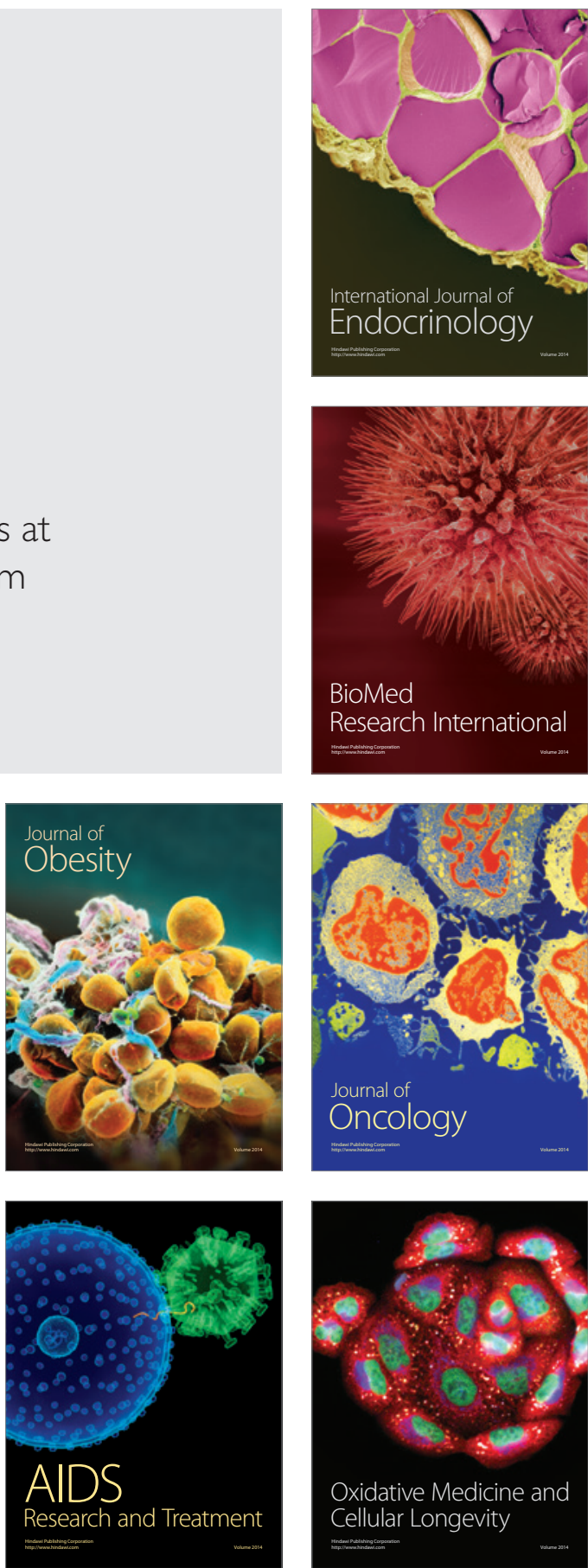